\section{Relapse of Experimental Autoimmune Encephalomyelitis after Discontinuation of FTY720 (Fingolimod) Treatment, but Not after Combination of FTY720 and Pathogenic Autoantigen}

\author{
Yuya Yoshide, ${ }^{a}$ Takumi Tsusi, ${ }^{a}$ Tetsuro FuJItA, ${ }^{b}$ and \\ Takeyuki KoHNO*,a,b
}

\begin{abstract}
${ }^{a}$ Department of Pathological Biochemistry, Faculty of Pharmaceutical Sciences, Setsunan University; 45-1 Nagaotoge-cho, Hirakata, Osaka 573-0101, Japan: and ${ }^{b}$ Research Institute for Production and Development; 15 Shimogamo, Morimoto-cho, Sakyo-ku, Kyoto 606-0805, Japan.

Received February 4, 2011; accepted March 19, 2011; published online April 8, 2011
\end{abstract}

FTY720 (Fingolimod) is known to have a significant therapeutic effect on experimental autoimmune encephalomyelitis (EAE). Here, we used an EAE mouse model, which had been established by immunizing $\mathrm{C} 57 \mathrm{BL} / 6 \mathrm{~J}$ mice with a partial peptide of myelin oligodendrocyte glycoprotein $\left(\mathrm{MOG}_{35-55}\right)$, to examine the relapse of EAE upon discontinuation of treatment with FTY720 alone or in combination with MOG $_{35-55}$. Relapse was confirmed to occur in all animals $(n=6)$ within one week after discontinuation of FTY720, with increase in the number of lymphocytes infiltrating the spinal cord and demyelination. However, in the case of combination therapy with FTY720 and MOG $_{35-55}$, relapse following discontinuation of treatment was completely suppressed. The autoantigenic peptide might serve to suppress the clonal selection of relapse-associated autoantigen-specific $T$ cells.

Key words FTY720; experimental autoimmune encephalomyelitis; multiple sclerosis; relapse

The novel immunomodulator FTY720 (Fingolimod) is a synthetic structural analogue of myriocin (ISP-I), a metabolite of Isaria cinclarii. ${ }^{1,2)}$ It was developed by Tetsuro Fujita, of our group, in collaboration with Taito Co. and Yoshitomi Pharmaceutical Industries, Ltd. in Japan, as a result of structural modification studies of ISP-I, aimed at reducing the toxicity of ISP-I, improving its physicochemical properties and identifying the essential structure for immunosuppressive activity (Fig. 1). ${ }^{3)}$ The efficacy of FTY720 has been well established in preclinical transplantation models (rat heart, liver, skin, small intestine, dog kidney and monkey kidney). ${ }^{4}$ Also, FTY720 has been reported to be effective in preventing the development of several immunological disease models, including rheumatoid arthritis, ${ }^{5)}$ myasthenia gravis, ${ }^{6}$ and atopic dermatitis. ${ }^{7)}$ The mechanism of action of FTY720 differs from that of established immunosuppressants, such as tacrolimus hydrate and cyclosporine. FTY720 is converted in vivo by sphingosine kinase to FTY720 monophosphate (FTY720-P), which is the active form of the drug. FTY720-P acts as a high-affinity agonist for sphingosine 1-phosphate (S1P) receptors. FTY720 blocks S1P signaling by inducing internalization and intracellular partial degradation of the receptors. ${ }^{8-10)}$ As a result, FTY720 suppresses immune response by sequestering circulating mature lymphocytes from blood and peripheral tissues to the secondary lymphoid tis-
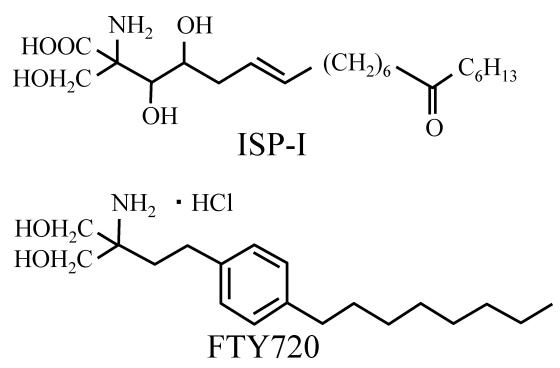

Fig. 1. Structures of ISP-I and FTY720 3 )

sues and thymus. ${ }^{11,12)}$

Experimental autoimmune encephalomyelitis (EAE) mice have been used as an animal model for human multiple sclerosis (MS) ${ }^{13)}$ In C57BL/6J mice, EAE is induced by immunization with a peptide consisting of residues 35-55 of myelin oligodendrocyte glycoprotein $\left(\mathrm{MOG}_{35-55}\right)$, a myelin sheath partial peptide. At an early stage of disease, stimulated helper $\mathrm{T}$ cells secrete considerable amounts of interleukin-17 (IL-17) and interferon- $\gamma$ (IFN- $\gamma$ ), representative cytokines produced by Th17 and Th1 cells. Consequently, the myelin sheath is damaged, resulting in nerve damage. It has already been demonstrated that FTY 720 has a significant therapeutic effect on EAE, ${ }^{14-16)}$ and FTY720 also reduced the number of lesions detected on MRI and the progression of clinical disease in patients with MS. ${ }^{17,18)}$ Recently, FTY720 has been approved by the Food and Drug Administration (U.S.A.) for treatment of MS. However, there has not yet been any report on the effect of discontinuation of FTY720 treatment in human MS or mouse EAE. Since FTY720 has no suppressive effect on activated lymphocytes, there may be a significant risk of relapse due to release of activated autoantigen-specific $\mathrm{T}$ cells from secondary lymphoid tissues to the peripheral tissues after discontinuation of FTY720.

In this study, we found that relapse occurred after discontinuation of FTY720 in EAE mice, whereas relapse did not occur after discontinuation of combination therapy with FTY720 and the autoantigenic peptide $\mathrm{MOG}_{35-55}$.

\section{MATERIALS AND METHODS}

Animals and Ethics C57BL/6J mice (8-week-old females) bred under specific pathogen-free conditions were purchased from Charles River Japan Inc. (Yokohama, Japan). The mice were given $\gamma$-ray-irradiated food (CRF-1, Oriental Bio Co., Kyoto, Japan) and distilled water for injection (Ohtsuka Pharmaceutical Co., Ltd., Tokyo, Japan). This study was performed according to a protocol approved by the institutional animal care committee of Setsunan University.

Agents 2-Amino-2-[2-(4-octylphenyl)ethyl]propane-1,3diol hydrochloride (FTY720 (Fingolimod)) was kindly provided by Yoshitomi Pharmaceutical Industries, Ltd., Japan. Cyclosporine (Sandimmun ${ }^{\circledR}$ for intravenous infusion) was purchased from Novartis Pharmaceuticals Corporation (Basel, Switzerland). Peptide 35(MEVGWYRSPFSRVVHLYRNGK)55 of myelin oligodendrocyte glycoprotein $\left(\mathrm{MOG}_{35-55}\right)$ was purchased from Operon Biotech. K. K. (AL, U.S.A.).

Induction of Experimental Autoimmune Encephalomyelitis C57BL/6J mice were immunized by subcutaneous injection of $\mathrm{MOG}_{35-55}(200 \mu \mathrm{g})$ with Freund's complete ad- 
juvant containing Mycobacterium tuberculosis $\mathrm{H} 37 \mathrm{Ra}$ (Difco, MI, U.S.A.) on the hind flanks. Pertussis toxin (200 ng; PT, List Biological Laboratories, Inc., CA, U.S.A.) was injected intravenously (i.v.) on days 0 and 2 . The clinical symptoms of EAE were checked daily and graded on a clinical score of $0-5$ : 0 , no clinical signs; 0.5 , partially limp tail; 1.0, paralyzed tail; 2.0 , loss of coordinated movement and hind limb paresis; 2.5 , one hind limb paralyzed; 3.0, both hind limbs paralyzed; 3.5, hind limbs paralyzed and weakness in forelimbs; 4.0, forelimbs paralyzed; 5.0, moribund. ${ }^{13)}$

Study Protocol I EAE mice were divided into two groups, 1) FTY720 group, given FTY720 in water $(0.3$ $\mathrm{mg} / \mathrm{kg}$, orally, once daily; this dosing regimen of FTY720 is similar to that reported by Kataoka et al. ${ }^{14)}$ ) from the period of onset (day 14 after the immunization) to the end of treatment (day 49 after the immunization), and 2) placebo group, given the vehicle (water) alone. Clinical symptoms of EAE and body weight were evaluated from the day of immunization to day 58. On days 14, 49 and 58 after the immunization, spinal cord was excised from mice under sodium pentobarbital anesthesia, and was examined histologically and immunohistochemically.

Study Protocol II EAE mice were treated from the time of onset of symptoms (day 14 after the immunization) with cyclosporine in water $(25 \mathrm{mg} / \mathrm{kg}$, orally, once daily; this dosing regimen of cyclosporine is similar to that reported by Foster et $a l .{ }^{16)}$ ) alone or in combination with FTY720 in water $(0.3 \mathrm{mg} / \mathrm{kg}$, orally, once daily). Clinical symptoms of EAE were evaluated from the day of immunization to day 58.

Study Protocol III EAE mice were treated from the time of onset of symptoms (day 14 after the immunization) with FTY720 in water $(0.3 \mathrm{mg} / \mathrm{kg}$, orally, once daily) alone or in combination with $\mathrm{MOG}_{35-55}$ in phosphate-buffered saline (PBS) $(1.0 \mu \mathrm{g} / \mathrm{mouse}$, i.v., once weekly). Treatment was terminated when the EAE score had improved to 0 or 0.5 ( 3 - 5 weeks), and then symptoms were evaluated up to 9 or $70 \mathrm{~d}$ after discontinuation of treatment.

Histochemical Staining On days 14, 49 and 58 after the immunization, following initial perfusion with PBS, EAE mice were perfused with $10 \%$ buffered formalin solution (Wako Pure Chemical Industries, Ltd., Osaka, Japan) and the spinal cord was removed. Tissues were processed, embedded in paraffin and cut into $5 \mu \mathrm{m}$ section. The sections were stained with hematoxylin-eosin (H\&E) using Mayer's Hematoxylin Soln. (Wako Pure Chemical Industries, Ltd.) or with

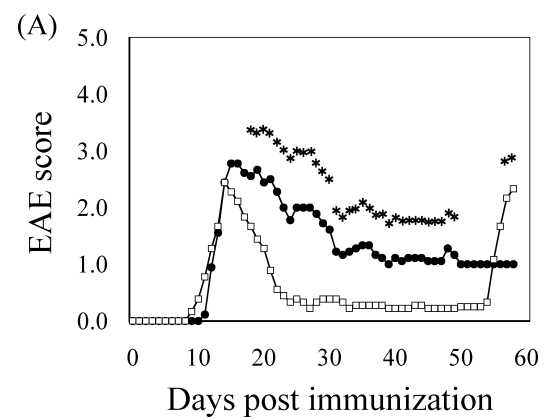

luxol fast blue (LFB) using Luxol ${ }^{\circledR}$ Fast Blue MSBN (Acros Organics, Geel, Belgium), or with goat anti-mouse CD3 monoclonal antibody (mAb) (Santa Cruz Biotechnology, Inc., CA, U.S.A.). The number of $\mathrm{CD}^{+}$cells ( $\mathrm{T}$ cells) was counted under a microscope with an eyepiece squared micrometer at a magnification of $\times 400$.

Statistical Analysis The significance of differences in EAE score, body weight and number of infiltrating $\mathrm{CD}^{+}$ cells (T cells) was evaluated by using the Mann-Whitney $U$ test.

\section{RESULTS}

Ameliorating Effect of FTY720 on Experimental Autoimmune Encephalomyelitis and Relapse after Discontinuation of Treatment EAE mice, which had been established by immunizing C57BL/6J mice with $\mathrm{MOG}_{35-55}$, were used to examine whether or not relapse occurs after discontinuation of FTY720 (Fingolimod). EAE mice $(n=12)$ were divided into an FTY720 group $(n=6)$, given FTY720 in water $(0.3 \mathrm{mg} / \mathrm{kg}$, orally, once daily), and a placebo group $(n=6)$, given the vehicle (water) alone (Study Protocol I). EAE score and body weight from the day of the immunization to day 58 are shown in Fig. 2. The EAE-associated symptoms were maintained in the placebo group, whereas they were significantly improved in the FTY720 group. However, relapse occurred about one week after discontinuation of FTY720 in all animals $(n=6)$ of the FTY720 group (Fig. 2A). The decrease in the body weight was correlated to the increase in the EAE score (Fig. 2B), and no effect of FTY720 on the body weight was observed.

To examine the infiltration of lymphocytes and the extent of demyelination, paraffin-embedded spinal cord, which had been obtained from a separate group under Study Protocol I, was stained with hematoxylin-eosin (H\&E) or luxol fast blue (LFB), or anti-mouse CD3 mAb. First, the infiltration of lymphocytes was examined by means of H\&E staining. Infiltration was observed at the onset (day 14 after immunization) (Figs. 3D, E), and was clearly suppressed at the end of treatment (day 49 after immunization) (Figs. 3G, H). However, at day 58 (relapse), infiltration was observed again (Figs. 3J, K). Second, demyelination was examined by using LFB staining. Demyelination was observed at the onset (Fig. 3F), and the myelin sheath was restored at the end of treatment (Fig. 3I). However, at relapse (day 58), demyelination was observed

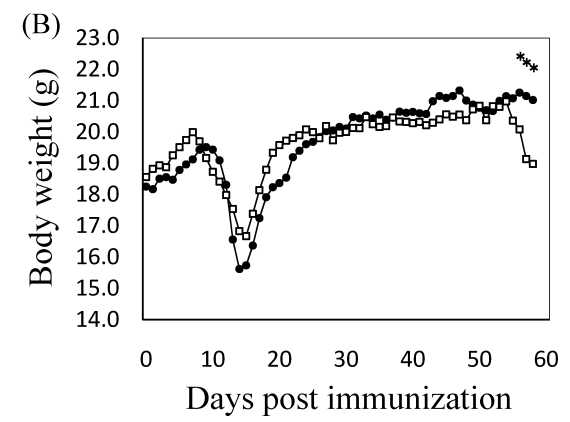

Fig. 2. Ameliorating Effect of FTY720 on Experimental Autoimmune Encephalomyelitis and Relapse after Discontinuation of FTY720

Experimental autoimmune encephalomyelitis (EAE) mice were divided into two groups, $\square$ : the FTY720 group ( $n=6$, FTY720 0.3 mg/kg, orally, once daily), $\bullet$ : the placebo group ( $n=6$, vehicle) (Study Protocol I). FTY720 was administered orally to EAE mice every day from day 14 after immunization for $35 \mathrm{~d}$ and EAE score (A) and body weight (B) were evaluated. The results are indicated as the mean values. The significance of differences in EAE score between the FTY720 group and the placebo group was examined by using the Mann-Whitney $U$-test $(*$ denotes $p<0.05)$. 


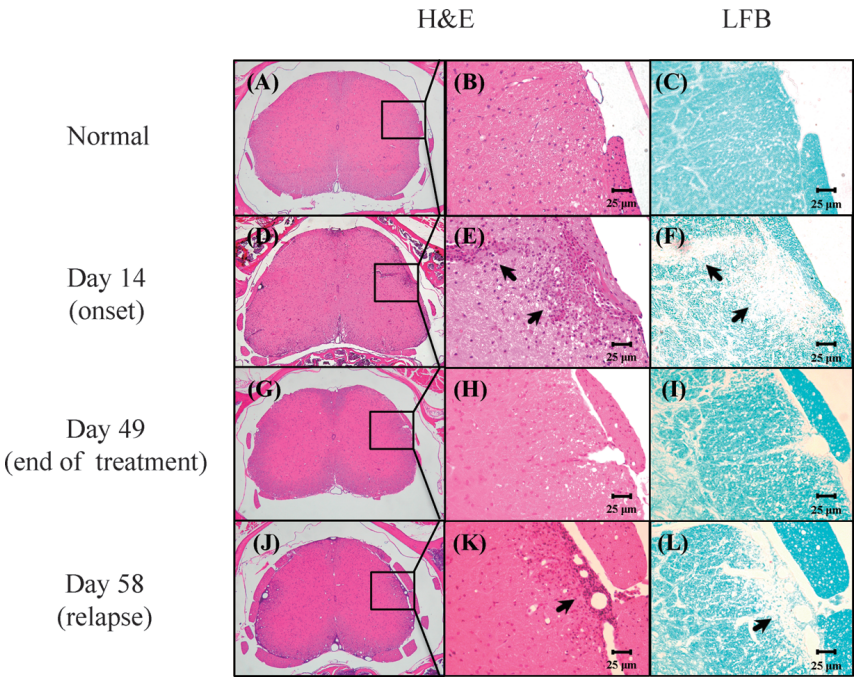

Fig. 3. Histochemical Staining of Spinal Cord

Experimental autoimmune encephalomyelitis mice were orally given FTY720 in water $(0.3 \mathrm{mg} / \mathrm{kg})$ daily from day 14 after immunization for $35 \mathrm{~d}$ (Study Protocol I). On days 14,49 and 58, spinal cord was excised and sections were stained with hematoxylin-eosin (H\&E) and luxol fast blue (LFB). (A-C): normal mice; (D-F): at day 14 (onset); $(\mathrm{G}-\mathrm{I})$ : at day 49 (end of treatment); (J-L): at day 58 (relapse). (A, B, D, E, G, H, J, K): H\&E; (C), (F), (I), (L): LFB. Representative images from one mouse in each day are shown. Infiltration of lymphocytes and demyelination were observed at days 14 and 58 (arrows).

again (Fig. 3L). To identify the cells that had infiltrated the spinal cord, CD3 staining was performed. The infiltration of $\mathrm{CD}^{+}$cells (T cells) per unit area in spinal cord is shown in Fig. 4. At the end of treatment, the number of $\mathrm{T}$ cells was significantly decreased to $4.0 \pm 3.2$ (S.D., $n=6)$ cells $/ \mathrm{mm}^{2}$ from $62.3 \pm 21.1$ (S.D., $n=6$ ) cells $/ \mathrm{mm}^{2}$ (at onset) in the FTY720 group. However, at relapse (day 58), it was significantly increased again to $59.5 \pm 26.7$ (S.D., $n=6$ ).

To examine whether or not the relapse after the discontinuation is FTY720-specific phenomenon, EAE mice were treated with cyclosporine (Study Protocol II). Though the EAE-associated symptoms became chronic, no fulminant course of the symptoms was observed (Fig. 5). The relapse after the discontinuation of FTY720 was not prevented by combination therapy with FTY720 and cyclosporine (Fig. 5).

Relapse of Experimental Autoimmune Encephalomyelitis on Discontinuation of Combination Therapy with FTY720 and MOG $_{35-55}$ EAE mice were used to examine whether or not relapse occurs following discontinuation of combination therapies with FTY720 and $\mathrm{MOG}_{35-55}$. EAE mice were treated from onset with FTY720 in water $(0.3$ $\mathrm{mg} / \mathrm{kg}$, orally, once daily) alone or in combination with $\mathrm{MOG}_{35-55}$ in phosphate-buffered saline $(1.0 \mu \mathrm{g} /$ mouse, i.v., once weekly) (Study Protocol III). Both treatments significantly improved the EAE-associated symptoms (Fig. 6). However, relapse was observed about one week after discontinuation of treatment in all mice of the FTY720 alone group. However, in the FTY720 plus $\mathrm{MOG}_{35-55}$ group, no relapse was observed in any of the animals $(n=6)$ following discontinuation (Fig. 6).

\section{DISCUSSION}

The novel immunomodulator FTY720 (Fingolimod) has

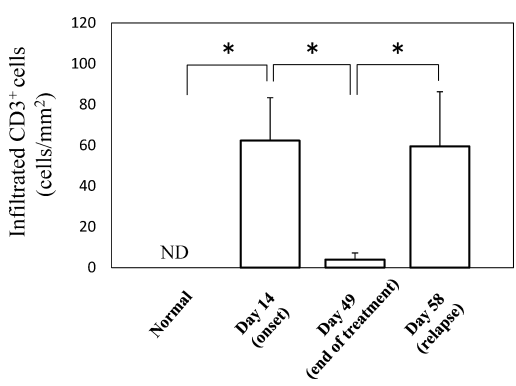

Fig. 4. Infiltrated $\mathrm{CD}^{+}$Cells in Spinal Cord

Spinal cord was excised as described in Fig. 3, and sections were stained with antimouse CD3. The number of $\mathrm{CD}^{+}$cells ( $\mathrm{T}$ cells) was counted under a microscope with an eyepiece squared micrometer at a magnification of $\times 400$. The results are indicated as the mean \pm S.D. of six animals and the significance of differences in the number was examined by using the Mann-Whitney $U$-test $(*$ denotes $p<0.05)$.

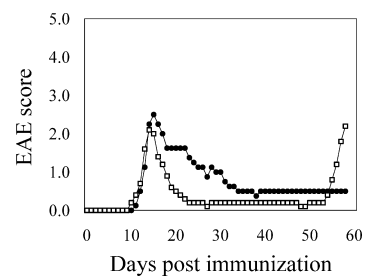

Fig. 5. Effect of Treatment Discontinuation on Relapse of Experimental Autoimmune Encephalomyelitis in the Case of Combination Therapy with FTY720 and Cyclosporine

Experimental autoimmune encephalomyelitis (EAE) mice were divided into two groups, $\square$ : the combination group $(n=5$, FTY $7200.3 \mathrm{mg} / \mathrm{kg}$, orally, once daily and cyclosporine $25 \mathrm{mg} / \mathrm{kg}$, orally, once daily), and $\mathbf{0}$ : the cyclosporine group ( $n=4$, cyclosporine $25 \mathrm{mg} / \mathrm{kg}$, orally, once daily) (Study Protocol II). The results are indicated as the mean values.

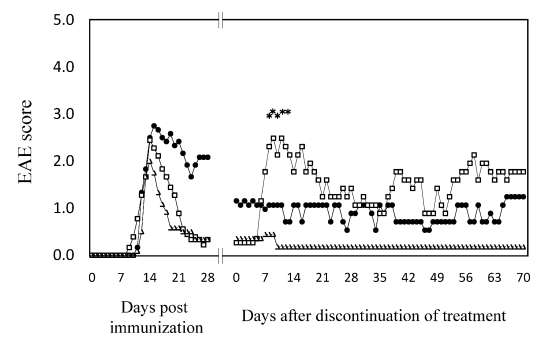

Fig. 6. Effect of Treatment Discontinuation on Relapse of Experimental Autoimmune Encephalomyelitis in the Case of Combination Therapy with FTY720 and $\mathrm{MOG}_{35-55}$

Experimental autoimmune encephalomyelitis (EAE) mice were treated from symptomatic onset with FTY720 alone or in combination with $\mathrm{MOG}_{35-55}$ (Study Protocol III). The treatments were discontinued when the EAE score had improved to 0 or 0.5 (3-5 weeks), and then symptoms were evaluated up to $9 \mathrm{~d}(n=6)$ or $70 \mathrm{~d}(n=3)$ after discontinuation of treatment. $\triangle$ : combination of FTY720 $+M O_{35-55}($ FTY720, $0.3 \mathrm{mg} / \mathrm{kg}$, orally, once daily and $\mathrm{MOG}_{35-55} 1.0 \mu \mathrm{g}$ /mouse, i.v., once weekly), $\square$ : FTY720 group (FTY720, $0.3 \mathrm{mg} / \mathrm{kg}$, orally, once daily), and $\mathbf{0}$ : the placebo group (vehicle). The results are indicated as the mean values. The significance of differences in EAE scores between the FTY720 group and the combination FTY720+MOG ${ }_{35-55}$ group was examined by using the Mann-Whitney $U$-test $(*$ denotes $p<0.05)$

a number of attractive properties. The mechanism of its immunosuppressive effect is different from those of established immunosuppressants, such as tacrolimus hydrate, cyclosporine, azathioprine, and mycophenolate mofetil. FTY720 suppresses immune response by sequestering circulating mature lymphocytes from blood and peripheral tissues to the secondary lymphoid tissues and thymus, rather than destruction of lymphocytes. At therapeutic doses, FTY720 does not affect $\mathrm{T}$ cell and $\mathrm{B}$ cell responses in vitro or in 
vivo. ${ }^{4,7)}$ Since FTY720 treatment allows preservation of many aspects of immune function, including the total number of lymphocytes, the capacity for lymphocyte activation in lymph nodes and tissues, the capacity for generating antibodies, and innate immune responses, ${ }^{19,20)}$ there is only a limited increase in susceptibility to infectious disease, including herpesvirus infection, urinary tract infection, and so on. ${ }^{21)}$ Furthermore, immune memory function is not impaired. ${ }^{4)}$

FTY720 ameliorates human multiple sclerosis (MS) and EAE-associated symptoms by sequestering circulating mature lymphocytes from blood and spinal cord to secondary lymphoid tissues and thymus. ${ }^{14-18)}$ However, there is no report on the effect of discontinuation of FTY720 treatment in human MS or mouse EAE. Our present results confirm that oral administration of FTY720 significantly improves EAEassociated symptoms. Infiltration of lymphocytes and demyelination in the spinal cord were suppressed by FTY720. However, one week after discontinuation of FTY720, lymphocyte infiltration and demyelination in the spinal cord reoccurred. When EAE was treated with cyclosporine alone, though the EAE-associated symptoms became chronic, no fulminant course of the symptoms was observed. Therefore, the relapse after discontinuation was a FTY720-specific phenomenon. These results suggested that $\mathrm{MOG}_{35}-55^{-}$-specific $\mathrm{T}$ cells might have been released from secondary lymphoid tissues and reactivated in the spinal cord. Since the amount of autoantigen that leaked from the spinal cord into the lymphoid system might have been reduced with the improvement of EAE, we speculate that there would be selective pressure favoring high-affinity pathogenic $\mathrm{T}$ cells. Thus, we considered that clonal selection of $\mathrm{MOG}_{35-55}$-specific $\mathrm{T}$ cells would have occurred in the secondary lymphoid tissues, and might be associated with the relapse. In contrast, it has already been demonstrated that long-term administration of FTY720 alone improved the relapse rate and risk of disability progression in human MS. ${ }^{21)}$ For this reason, when EAE (and probably MS) is treated with FTY720 alone, administration for a sufficiently long period to completely block leakage of autoantigen(s) from the spinal cord would be necessary to induce complete remission.

In this study, we demonstrated that combination therapy with FTY720 and MOG $_{35-55}$ completely suppressed the relapse upon discontinuation of treatment; that is, though helper $\mathrm{T}$ cells returned to the peripheral blood (data not shown), no relapse was observed. In contrast, relapse was not prevented by combination therapy with FTY720 plus cyclosporine, that is, by sequestering circulating mature lymphocytes to secondary lymphoid tissues (FTY720) and suppressing $\mathrm{T}$ cell function (cyclosporine). Thus, the combination therapy with FTY720 and $\mathrm{MOG}_{35-55}$ might not only inhibit immune response, but also induce immune tolerance, possibly by suppressing the clonal selection of $\mathrm{MOG}_{35-55^{-}}$ specific $\mathrm{T}$ cells. The remission-induction mechanism(s) of $\mathrm{MOG}_{35-55}$ might be similar to that of glatiramer acetate (GA). GA is a random polymer of glutamic acid, lysine, alanine, and thyrosine, and the amino acid composition of GA is similar to that of myelin basic protein (MBP). ${ }^{22)} \mathrm{GA}$ is thought to act as an altered peptide ligand and to inhibit acti- vation of MBP-specific $\mathrm{T}$ cell clones in vitro. ${ }^{22,23)}$ Thus, the next step will be to establish in detail the mechanism(s) through which clonal selection of autoreactive $\mathrm{T}$ cells is prevented by combination therapy with FTY720 and autoantigen.

In conclusion, the results of this study suggest that combination therapy with FTY720 and autoantigen may become a breakthrough remission-induction therapy for MS.

\section{REFERENCES}

1) Fujita T., Inoue K., Yamamoto S., Ikumoto T., Sasaki S., Toyama R., Chiba K., Hoshino Y., Okumoto T., J. Antibiot., 47, 208-215 (1994).

2) Kiuchi M., Adachi K., Kohara T., Minoguchi M., Hanano T., Aoki Y., Mishina T., Arita M., Nakao N., Ohtsuki M., Hoshino Y., Teshima K., Chiba K., Sasaki S., Fujita T., J. Med. Chem., 43, 2946-2961 (2000).

3) Matsumoto N., Hirose R., Sasaki S., Fujita T., Chem. Pharm. Bull., 56, 595-597 (2008).

4) Brinkmann V., Pinschewer D., Chiba K., Feng L., Trends Pharmacol. Sci., 21, 49-52 (2000).

5) Tsunemi S., Iwasaki T., Kitano S., Imado T., Miyazawa K., Sano H., Clin. Immunol., 136, 197-204 (2010).

6) Kohno T., Tsuji T., Hirayama K., Iwatsuki R., Hirose M., Watabe K., Yoshikawa H., Kohno T., Matsumoto A., Fujita T., Hayashi M., Biol. Pharm. Bull., 28, 736-739 (2005).

7) Kohno T., Tsuji T., Hirayama K., Watabe K., Matsumoto A., Kohno T., Fujita T., Biol. Pharm. Bull., 27, 1392-1396 (2004).

8) Brinkmann V., Davis M. D., Heise C. E., Albert R., Cottens S., Hof R., Bruns C., Prieschl E., Baumruker T., Hiestand P., Foster C. A., Zollinger M., Lynch K. R., J. Biol. Chem., 277, 21453-21457 (2002).

9) Matloubian M., Lo C. G., Cinamon G., Lesneski M. J., Xu Y., Brinkmann V., Allende M. L., Proia R. L., Cyster J. G., Nature (London), 427, 355-360 (2004).

10) Galer M. H., Goetzl E. J., FASEB J., 18, 551—553 (2004).

11) Chiba K., Yanagawa Y., Masubuchi Y., Kataoka H., Kawaguchi T., Ohtsuki M., Hoshino Y., J. Immunol., 160, 5037-5044 (1998).

12) Yanagawa Y., Sugahara K., Kataoka H., Kawaguchi T., Masubuchi Y., Chiba K., J. Immunol., 160, 5493-5499 (1998).

13) Stromnes I. M., Goverman J. M., Nat. Protoc., 1, 1810-1819 (2006).

14) Kataoka H., Sugahara K., Shimano K., Teshima K., Koyama M., Fukunari A., Chiba K., Cell. Mol. Immunol., 2, 439-448 (2005).

15) Papadopoulos D., Rundle J., Patel R., Marshall I., Stretton J., Eaton R., Richardson J. C., Gonzalez M. I., Philpott K. L., Reynolds R., J. Neurosci. Res., 88, 346-359 (2010).

16) Foster C. A., Howard L. M., Schweitzer A., Persohn E., Hiestand P. C., Balatoni B., Reuschel R., Beerli C., Schwartz M., Billich A., J. Pharmacol. Exp. Ther, 323, 469-475 (2007).

17) Kappos L., Antel J., Comi G., Montalban X., O’Connor P., Polman C. H., Haas T., Korn A. A., Karlsson G., Radue E. W., FTY720 D2201 Study Group, N. Engl. J. Med., 355, 1124-1140 (2006).

18) Fox E. J., Am. J. Manag. Care, 16, 219-226 (2010).

19) Cohen J. A., Barkhof F., Comi G., Hartung H. P., Khatri B. O., Montalban X., Pelletier J., Capra R., Gallo P., Izquierdo G., Tiel-Wilck K., de Vera A., Jin J., Stites T., Wu S., Aradhye S., Kappos L., TRANSFORMS Study Group, N. Engl. J. Med., 362, 402-415 (2010).

20) Mehling M., Brinkmann V., Antel J., Bar-Or A., Goebels N., Vedrine C., Kristofic C., Kuhle J., Lindberg R. L., Kappos L., Neurology, 71, 1261-1267 (2008)

21) Kappos L., Radue E. W., O’Connor P., Polman C., Hohlfeld R., Calabresi P., Selmaj K., Agoropoulou C., Leyk M., Zhang-Auberson L., Burtin P., FREEDOMS Study Group, N. Engl. J. Med., 362, 387-401 (2010).

22) Racke M. K., Lovett-Racke A. E., J. Immunol., 186, 1887-1890 (2011).

23) Aharoni R., Teitelbaum D., Arnon R., Sela M., Proc. Natl. Acad. Sci. U.S.A., 96, 634-639 (1999). 